\title{
A criteria-based rehabilitation program for chronic mid-portion Achilles tendinopathy: study protocol for a randomised controlled trial
}

Colin Griffin ${ }^{1,2^{*}}$ (D), Katherine Daniels ${ }^{2,3,4}$, Caroline Hill ${ }^{2}$, Andrew Franklyn-Miller ${ }^{2,5}$ and Jean-Benoit Morin ${ }^{1,6,7}$

\begin{abstract}
Background: Achilles tendinopathy (AT) is a common overuse injury in running-related sports where patients experience pain and impaired function which can persist. A graded rehabilitation program has been successful in reducing pain and improving function to enable a return to sport. The aim of this study is to compare the effectiveness of a criteria-based rehabilitation program including strength and reactive strength targets, with a previously successful rehabilitation program on changes in pain and function using the Victorian Institute of Sport Assessment-Achilles (VISA-A) questionnaire. Secondary aims will be to assess changes in calf strength, reactive strength, and lower limb running and forward hop biomechanics over the course of a 12-week rehabilitation program, and long-term follow-up investigations.
\end{abstract}

Methods: Sixty eligible participants with chronic mid-portion AT who train in running-based sports will be included in this study. They will be randomly assigned to a group that will follow an evidence-based rehabilitation program of daily exercises with progression guided by symptoms or a group performing 3 high-intensity rehabilitation sessions per week with individualised load targets progressing to reactive strength exercises. Testing will take place at baseline, week 6 and 12. Plantar flexor peak torque will be measured using isokinetic dynamometry, reactive strength will be measured using a drop jump and lower limb biomechanical variables will be measured during a single leg forward hurdle hop test and treadmill running using 3D motion analysis. Follow-up interviews will take place at 6, 12 and 24 months after beginning the program which will assess patient participation in sport and possible re-injury.

Discussion: This is the first study to propose an individualised criteria-based graded rehabilitation program in patients in with chronic mid-portion Achilles tendinopathy where progression is guided by strength and reactive strength outcome measures. This study will provide a comprehensive assessment of plantar flexor strength, reactive strength and lower limb biomechanical variables in running and forward hopping with the VISA-A questionnaire as the primary outcome measure and long term post-intervention follow-up assessments performed.

Trial registration: ClinicalTrials.gov (ID: NCT04384874). Registered retrospectively on April 23rd 2020.

Keywords: Achilles tendinopathy, Plantar flexor strength, Stiffness, Hopping, Achilles tendon, Injury, Rehabilitation

\footnotetext{
* Correspondence: colingriffin@sportssurgeryclinic.com

'Université Côte d'Azur, LAMHESS, Nice, France

${ }^{2}$ Sports Surgery Clinic, Santry Demesne, Dublin 9, Ireland

Full list of author information is available at the end of the article
}

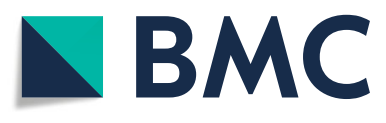

(c) The Author(s). 2021 Open Access This article is licensed under a Creative Commons Attribution 4.0 International License, which permits use, sharing, adaptation, distribution and reproduction in any medium or format, as long as you give appropriate credit to the original author(s) and the source, provide a link to the Creative Commons licence, and indicate if changes were made. The images or other third party material in this article are included in the article's Creative Commons licence, unless indicated otherwise in a credit line to the material. If material is not included in the article's Creative Commons licence and your intended use is not permitted by statutory regulation or exceeds the permitted use, you will need to obtain permission directly from the copyright holder. To view a copy of this licence, visit http://creativecommons.org/licenses/by/4.0/ The Creative Commons Public Domain Dedication waiver (http://creativecommons.org/publicdomain/zero/1.0/) applies to the data made available in this article, unless otherwise stated in a credit line to the data. 


\section{Background}

The Achilles tendon is the largest and strongest tendon in the human body [1] and usually withstands very high tensile forces during exercise [2], but is also one of the most commonly injured tendons [3]. Achilles tendinopathy (AT) affects $2 \%$ of the general population [4], and has an incidence of 7-9\% in running-based sports with a cumulative lifetime incidence of up to $52 \%$ among certain athletic populations [5].

Tendinopathy is described as pain and impaired function in the affected tendon [6-8]. Over time this may result in reduced physical activity, absenteeism from sport and impaired quality of life [8]. Histologically and biochemically pathological tendon has been shown to include increased hyper-cellularity, reduced collagen type I and increased type III content, increased proteoglycans and glycosaminoglycans, and blood vessel in-growth $[9,10]$. Excessive loading of the tendon is believed to be the primary contributory factor to Achilles tendinopathy [11]. The fibroblastic cells known as tenocytes within the extracellular matrix are sensitive to mechanical loading and, when the tendon is overloaded, the cells alter the protein composition of the matrix resulting in pathology and reduced capacity for exercise [12].

Patients with AT usually present with pain, swelling and impaired performance of the tendon [13], as well as altered function of the plantar flexor muscles [14-16]. In a sporting population, training load perturbations such as a rapid increase in training volume, intensity or frequency are said to be common contributory factors [17]. Re-injury rates are high, most likely due to incomplete restoration of muscle-tendon unit function [18], and symptoms can persist for a number of years in some cases [19]. In an eight-year follow-up study, $20 \%$ of patients still experienced impaired physical activity [20]. A failed healing response and degenerative changes are associated with the development of chronic tendinopathy resulting in reduced load capacity and persistent pain symptoms [8]. This is described by Cook et al. [8] in their proposed continuum model involving three stages: reactive tendinopathy, tendon disrepair and degenerative tendinopathy.

Impairments in tendon mechanical properties such as stiffness and Young's modulus have been highlighted in AT [21-23]. Wang et al. (2012) observed reduced stiffness and increased hysteresis of the Achilles tendon, along with reduced rate of force development of the plantar flexor muscles and reduced single leg horizontal hop performance in symptomatic limbs of patients with AT, compared to the non-symptomatic limb. While one study found reduced lower limb stiffness in the injured limb of runners with AT during hopping [24], biomechanical variables such as leg and joint stiffness in running and hopping tasks have not been extensively researched in patients with Achilles tendinopathy.

Many passive treatment therapies such as injections $[25,26]$, Non-Steroidal Anti-Inflammatory Drugs (NSAI Ds) [10, 27], ultrasound [10, 27], shockwave [28, 29], laser [27], iontophoresis [25], acupuncture [30], orthotics $[25,29]$, wearing a night splint [31], vibration and cryotherapy [32], mucopolysaccharides [33], and a wait-andsee approach [28] have been used in the management of AT. However, there is strongest evidence for the use of exercise therapy as the primary treatment option [3436]. Tendons adapt to exercise as the mechanical perturbation of the inter- and intra-fascicular cells triggers a molecular response signalling an expression of important proteins in the extra-cellular matrix which restore the mechanical properties of the tendon [37, 38]. Three main modes of exercise have been widely used, each associated with improvements in clinical symptoms to varying degrees, namely: Alfredson's eccentric protocol [39], Silbernagel's combined concentric-eccentric protocol [36] and the Heavy Slow Resistance protocol [40]. The Silbernagel protocol [see Table 1] comprises a combined concentric-eccentric exercise program performed daily, before progressing to plyometric exercises as symptoms permit, with no individualisation of exercise prescription and progression guided solely by pain symptoms on a numeric pain rating scale (NPRS).

Recent evidence suggests that magnitude of loading, irrespective of contraction mode, is the primary stimulus for tendon adaptation [41]. Isometric exercises using $5 \times$ $45 \mathrm{~s}$ contractions at $70 \%$ maximal voluntary contraction (MVC) have been used for early management of tendinopathies with evidence suggesting an analgesic effect [42]. However, this has been since contested by the findings of O'Neill et al. [43]. Documented timeframes for rehabilitation interventions vary between 6 weeks to several months with no clear objective measures for return to sport. Patients with AT display impaired reactive strength qualities during hopping tasks and it is recommended to include plyometric training at an advanced stage of a rehabilitation program to prepare for the stretch-shortening cycle demands of running-based sports $[19,36]$. A multi-stage rehabilitation program that includes the combination of strength development and plyometric training may thus be beneficial, but there is a lack of consensus on assessing these qualities to guide exercise prescription and progression through the rehabilitation pathway.

While numerous studies have shown positive clinical improvements and tendon adaptations to exercise [36, 39-41, 44], studies which investigate a periodised return to sport rehabilitation program with load targets and outcome measures for progression, are necessary due to the individualised nature of its initial presentation and 
Table 1 Silbernagel's combined concentric-eccentric program

\section{Phase 1: Weeks 1-2}

Patient status: Pain and difficulty with all activities, difficulty performing 10 single leg heel raises

Goal: Start to exercise, gain understanding of their injury and of pain monitoring model

Treatment program: Perform exercises every day:

- Pain monitoring model information and advice on exercise activity

- Circulation exercises (moving foot up and down)

- Double leg heel raises standing on the floor $(3 \times 10-15$ repetitions)

- Single leg heel raises standing on the floor $(3 \times 10)$

- Sitting heel raises $(3 \times 10)$

- Eccentric heel raises standing on the floor $(3 \times 10)$

\section{Phase 2: Weeks 2-5}

Patient status: Pain and difficulty with all activities, difficulty performing 10 single leg heel raises

Goal: Start to exercise, gain understanding of their injury and of pain monitoring model

Treatment program: Perform exercises every day:

- Pain monitoring model information and advice on exercise activity

- Circulation exercises (moving foot up and down)

- Double leg heel raises standing on the floor (3×10-15 repetitions)

- Single leg heel raises standing on the floor $(3 \times 10)$

- Sitting heel raises $(3 \times 10)$

- Eccentric heel raises standing on the floor $(3 \times 10)$

Patient status: Pain with exercise, morning stiffness, pain when performing heel raises

Goal: Start strengthening

Treatment program: Perform exercises every day:

- Double leg heel raises standing on the edge of a step $(3 \times 15)$

- Single leg heel raises standing on the edge of a step $(3 \times 10)$

- Sitting heel raises $(3 \times 15)$

- Eccentric heel raises standing on the edge of a step $(3 \times 15)$

- Quick-rebounding heel raises $(3 \times 20)$

\section{Phase 3: Weeks 3-12 (longer if needed)}

Patient status: Tolerates phase 2 exercise program well, no pain at distal portion of tendon, possibly increased or decreased morning stiffness

Goal: Heavier strength training, increase or begin running and/or jumping

Treatment program: Perform exercises every day with heavier load 2-3 times per week

- Single leg heel raises standing on the edge of a step with added weight $(3 \times 10)$

- Sitting heel raises $(3 \times 15)$

- Eccentric heel raises standing on the edge of a step with added weight $(3 \times 15)$

- Quick-rebounding heel raises $(3 \times 20)$

- Plyometric training

Phase 4: Weeks 12-6 months (longer if needed)

Patient status: Minimal symptoms, morning stiffness but not every day, can participate in sports without difficulty

Goal: Heavier strength training, increase or begin running and/or jumping

Treatment program: Perform exercises every day with heavier load 2-3 times per week

- Single leg heel raises standing on the edge of a step with added weight $(3 \times 10)$

- Eccentric heel raises standing on the edge of a step with added weight $(3 \times 15)$

- Quick-rebounding heel raises $(3 \times 20)$ 


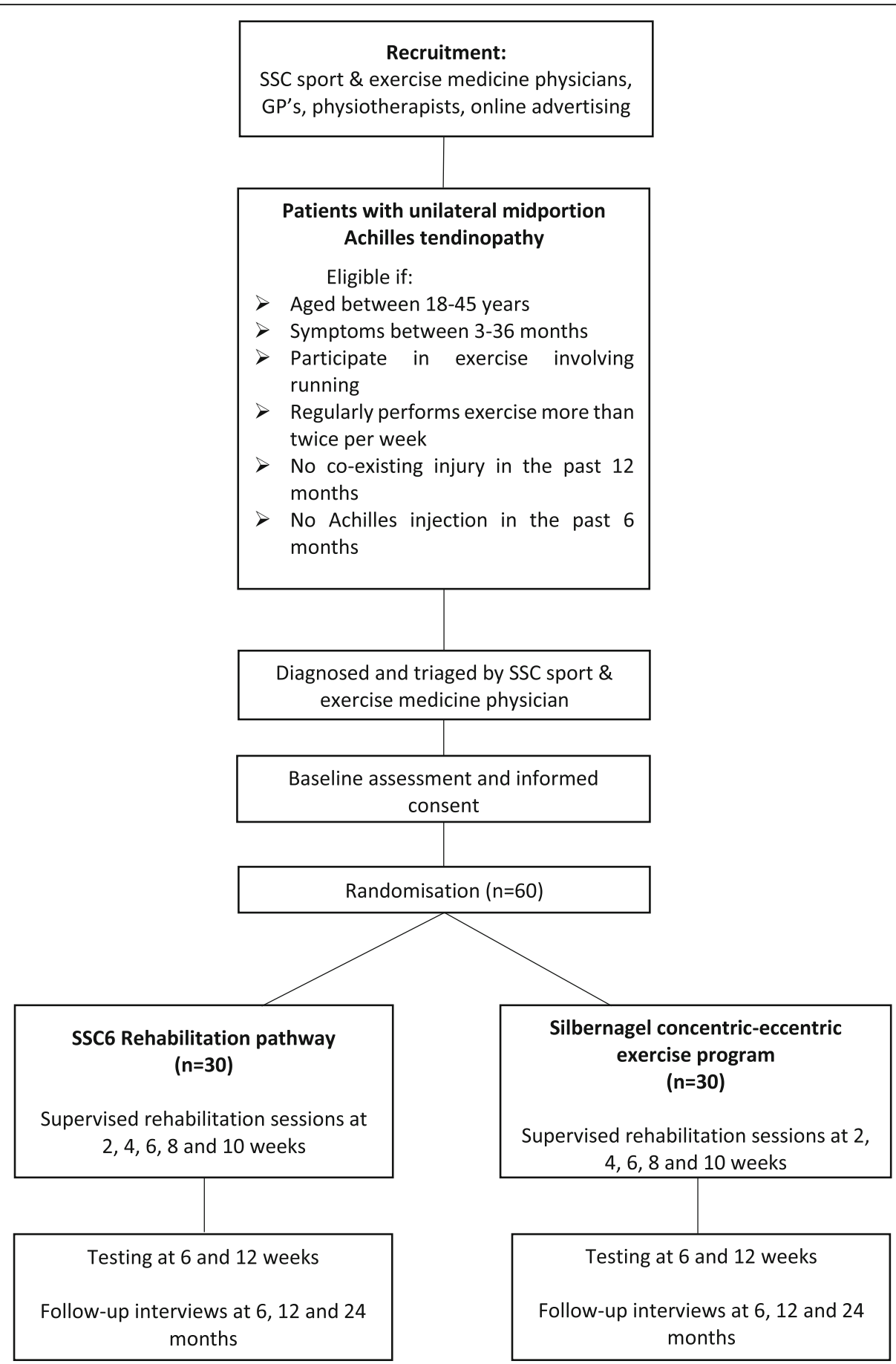

Fig. 1 Study design flow chart

diverse timeframes for recovery. The Sports Surgery Clinic (SSC) rehabilitation pathway [see Table 2] proposed in this study involves 6 stages of progressive rehabilitation (SSC6) from initial diagnosis and assessment, through developing strength, power and reactive strength, linear and multi-directional running, and return to performance. The existing literature has demonstrated positive clinical outcomes using Silbernagel's rehabilitation program [18, 44] and we have selected this a suitable comparative control for this study which involves a graded progression pathway from combined concentric-eccentric exercises to plyometric training [36].

Considering the multiple functional impairments experienced by athletes with $\mathrm{AT}$, a battery of kinematic and 
Table 2 SSC6 Rehabilitation program

\begin{tabular}{|c|c|c|c|}
\hline $\begin{array}{l}\text { Level } 1 \\
\text { Week } 0-3 \\
\text { Entry criteria: } \\
\text { Pain > 5/10 on SL calf raise }\end{array}$ & $\begin{array}{l}\text { Kinetic chain (2 } \\
\text { days per week) } \\
\text { Box squat } 3 \times 8 \\
\text { reps @ } 10 \mathrm{RM} \\
\text { Step-up } 3 \times 8 \text { reps } \\
\text { e/s @ 10RM }\end{array}$ & $\begin{array}{l}\text { Calf Isometrics (daily) } \\
5 \times 45 \text { s holds @ } 60 \text { s RM) }\end{array}$ & \\
\hline $\begin{array}{l}\text { Level } 2 \\
\text { Week } 0-4 \\
\text { Entry criteria: } \\
\text { Pain }<5 / 10 \text { on SL calf raise }\end{array}$ & $\begin{array}{l}\text { Kinetic chain (2 } \\
\text { days per week) } \\
\text { Front squat } 4 \times 8 \\
\text { reps @ 10RM } \\
\text { Or } \\
\text { Deadlift } 4 \times 8 \text { reps } \\
\text { @ } 10 \text { RM } \\
\text { Step-up } 3 \times 8 \text { e/s @ } \\
10 R M \\
\text { Or } \\
\text { Split squat } 3 \times 8 \\
\text { reps e/s }\end{array}$ & $\begin{array}{l}\text { Calf strength ( } 3 \text { days per week) } \\
\text { SL calf raises } 4 \times 8 \text { reps @ 10RM } \\
\sim \text { Begin with dumbbell and shoes off } \\
\sim \text { Aim to through 1st MTPJ and good } \\
\text { rearfoot control } \\
\text { Seated SL calf raises } 4 \times 10 \text { reps @ } \\
12 \mathrm{RM} \\
\sim \text { Begin with kettlebell on knee and } \\
\text { forefoot on a plate }\end{array}$ & \\
\hline $\begin{array}{l}\text { Level } 3 \\
\text { Week } 3-6 \\
\text { Entry criteria: } \\
\text { Pain }<4 / 10 \text { on SL calf raise }\end{array}$ & $\begin{array}{l}\text { Kinetic chain (2 } \\
\text { days per week) } \\
\text { Front squat } 4 \times 6 \\
\text { reps @ 8RM } \\
\text { Or } \\
\text { Deadlift } 4 \times 6 \text { reps } \\
\text { @ 8RM } \\
\text { Step-up } 3 \times 6 \text { e/s @ } \\
8 R M \\
\text { Or } \\
\text { Split squat } 3 \times 6 \\
\text { each side @ 8RM }\end{array}$ & $\begin{array}{l}\text { Calf strength (3 times per week) } \\
\text { SL calf raises } 4 \times 8 \text { reps @ 10RM } \\
\sim \text { Progress to a smith machine or } \\
\text { barbell using rack for support } \\
\sim \text { Aim for }>70 \% \text { BW } \\
\text { Seated SL calf raises } 4 \times 10 \text { reps @ } \\
\text { 12RM } \\
\sim \text { Progress to smith machine or } \\
\text { landmine press } \\
\sim \text { Aim for }>90 \% \text { BW }\end{array}$ & $\begin{array}{l}\text { Coordination/running } \\
\text { technique } \\
\text { Ankling } 3 \times 10 \mathrm{~m} \\
\text { March } 3 \times 10 \mathrm{~m} \\
\text { A-skip } 3 \times 20 \mathrm{~m}\end{array}$ \\
\hline $\begin{array}{l}\text { Level } 4 \\
\text { Week 6-9 } \\
\text { Entry criteria: } \\
\text { Pain }<5 / 10 \text { for } 10 \text { DL hops } \\
<10 \% \text { asymmetry in calf } \\
\text { isokinetic tests }\end{array}$ & $\begin{array}{l}\text { Kinetic chain (2 } \\
\text { days per week) } \\
\text { Front squat } 4 \times 6 \\
\text { reps @ 8RM } \\
\text { Or } \\
\text { Deadlift } 4 \times 6 \text { reps } \\
\text { @ 8RM } \\
\text { Step-up } 3 \times 6 \text { reps } \\
\text { e/s @ 8RM } \\
\text { Or } \\
\text { Split squat } 3 \times 6 \\
\text { each side @ 8RM }\end{array}$ & $\begin{array}{l}\text { Calf strength (3 times per week) } \\
\text { SL calf raises } 4 \times 8 \text { reps @ 10RM } \\
\sim \text { Progress to a smith machine or } \\
\text { barbell using rack for support } \\
\sim \text { Aim for }>80 \% \text { BW } \\
\text { Seated SL calf raises } 4 \times 10 \text { reps @ } \\
\text { 12RM } \\
\sim \text { Progress to smith machine or } \\
\text { landmine press } \\
\sim \text { Aim for }>110 \% \text { BW }\end{array}$ & $\begin{array}{l}\text { DL Reactive strength }(2 \\
\text { times per week) } \\
\text { DL Pogo hops in-place } 4 \times \\
10 \text { (Day } 1 \text { ) } \\
\sim \text { Keep knees straight and } \\
\text { stiff } \\
\sim \text { Flat foot contacts } \\
\sim \text { Active dorsiflexion during } \\
\text { flight phase } \\
\text { DL pogo hops forward } 4 \times \\
10 \text { (Day } 2 \text { ) } \\
\sim \text { Keep knees straight and } \\
\text { stiff } \\
\sim \text { Flat foot contacts } \\
\sim \text { Active dorsiflexion during } \\
\text { flight phase }\end{array}$ \\
\hline
\end{tabular}

Level 5

Week 9-12

Entry criteria:

Pain < 4/10 for 10 SL hops

Exit criteria:

$<10 \%$ asymmetry in single leg vertical and horizontal RSI

Level 6

Week 12-26

Recommended maintenance program
Kinetic chain (2 Calf strength (3 times per week)

days per week) SL calf eccentric $4 \times 8$ reps @ 10RM Front squat $3 \times 5$ reps@7RM Or

Deadlift $3 \times 5$ reps @ 7RM

Step-up $3 \times 5$ reps e/s@7RM Or

Split squat $3 \times 5$ each side @ 7RM

Kinetic chain ( 2 days per week) Front squat $3 \times 5$ reps@ $@ 7 R M$ Or

Deadlift $3 \times 5$ reps @ 7RM

Step-up $3 \times 5$ reps

e/s@7RM $\sim$ Use a smith machine/leg press/ barbell using rack for support

$\sim$ Up on 2 legs, lower down slowly on 1 over $3 \mathrm{~s}$

$\sim$ Aim for $>100 \%$ BW or equivalent Seated SL calf raises 4×10 reps @ $12 \mathrm{RM}$

$\sim$ Progress to smith machine or landmine press

Aim for $>110 \%$ BW

Calf strength (2 times per week) $\mathrm{SL}$ calf isometric $4 \times 8$ reps $\times 4 \mathrm{~s}$ holds

$\sim$ Use a smith machine/leg press/ barbell using rack for support

$\sim$ Up on 2 legs, hold on 1

$\sim$ Aim for $>140 \%$ BW or equivalent

\section{Reactive strength (2}

times per week)

Drop jump $4 \times 4$ reps from 20 to $30 \mathrm{~cm}$ box

$\sim$ Maximum jump height with minimal contact

- Minimal knee bend on ground contact

Cue "imagine the floor is hot"

DL Reactive strength (2 times per week) Drop jump $4 \times 5$ reps from 20 to $30 \mathrm{~cm}$ box

(2 times per week)

pogo hops in-place $4 \times 12 \mathrm{e} / \mathrm{s}$ (Day 1 ) SL pogo hops forward $4 \times 12 \mathrm{e} / \mathrm{s}$ (Day 2)
SL Reactive strength (2 times per week) × $10 \mathrm{e} / \mathrm{s}$ (Day 1) $4 \times 10 \mathrm{e} / \mathrm{s}$ (Day 2) SL pogo hops forward 
Table 2 SSC6 Rehabilitation program (Continued)

\begin{tabular}{lll}
\hline Level 1 & Kinetic chain (2 & Calf Isometrics (daily) \\
Week $0-3$ & days per week) & $5 \times 45$ s holds @ 60 s RM) \\
Entry criteria: & Box squat $3 \times 8$ & \\
Pain $>5 / 10$ on SL calf raise & $\begin{array}{l}\text { reps @ 10RM } \\
\text { Step-up } 3 \times 8 \text { reps } \\
\text { e/s @ 10RM }\end{array}$ \\
\hline & Or \\
& Split squat 3 $\times 5$ \\
& each side @ 7RM \\
\hline
\end{tabular}

Abbreviations: DL - double leg, SL - single leg, reps - repetitions, e/s - each side, BW - bodyweight, RM - repetition maximum

kinetic tests to investigate plantar flexor strength, reactive strength and lower limb biomechanical variables in hopping and running, may provide guidance on exercise prescription, progression through a rehabilitation program and return to sport decision-making. To the best of our knowledge no study has assessed such breadth of athletic qualities affected by AT.

This study will aim to compare the outcome of SSC6, a multi-factorial, individualised criteria-based rehabilitation program with Silbernagel's combined concentriceccentric program, in physically active participants with chronic mid-portion AT. In addition to the commonly reported outcome measures of VISA-A, as secondary outcome measures we will assess plantar flexor strength, reactive strength and lower limb kinematic and kinetics during running and hopping at 6-week intervals during a 12-week rehabilitation program as these have not been reported previously. We also further aim to investigate the long term effects of rehabilitation programs and achieved outcome measures over a 6, 12 and 24-month follow-up period.

\section{Aims}

Using the VISA-A questionnaire as the primary outcome measure, the aim of this study is to compare the effectiveness of Silbernagel's daily exercise program with progression guided by pain symptoms, against SSC6's exercise program carried out 3 times per week with specific load targets. We will assess plantar flexor strength using isokinetic dynamometry, reactive strength based on a drop jump, and lower limb biomechanics during a novel single leg horizontal rebound test and running, and investigate whether changes in these variables over the course of the 12-week rehabilitation program are associated with improved pain and function outcomes using the VISA-A questionnaire when comparing the two rehabilitation programs. We will assess participant satisfaction with their prescribed program, adherence and fidelity using a training diary and perform follow-up interviews at 6, 12 and 24 months to analyse participation in their sport and any potential re-injury rates.

\section{Methods \\ Study design}

This study will be a single-centre, parallel group randomized-control trial. The data collection will take place at the SSC Sports Medicine department at the Sports Surgery Clinic in Dublin. The study protocol has been reported using the Standard Protocol Items: Recommendations for Interventions (SPIRIT) statement guidelines [Available in supplementary files]. The study was approved by the Sports Surgery Clinic's Research Ethics Committee, (Application number: SAREB13/05/ 19CG/MJ) and registered at ClinicalTrials.gov (ID: NCT04384874).

\section{Participants}

Adult patients diagnosed with chronic mid-portion AT who participate in running-based sports will be invited to take part in this study. Patients who present to the Sports Surgery Clinic (SSC) with Achilles pain will be seen by a Sport and Exercise medicine physician, their history and clinical examination will be confirmed with magnetic resonance imaging (MRI). If the patient is diagnosed and meets the inclusion criteria, they will be invited to participate in the study and will be given an information sheet to read with a minimum of $24 \mathrm{~h}$ to consider before agreeing by signing a consent form [Additional file 2: Appendix 2]. Participants will also be recruited externally through adverts on social media channels, emails to coaching contacts and local sports clubs. Participants who feel that they are eligible and meet the inclusion criteria will be referred for examination by a sport and exercise medicine physician at the clinic to confirm diagnosis and eligibility for the study.

\section{Inclusion/exclusion criteria Inclusion criteria}

Participants will be eligible for this study if they are aged 18-45 years, perform running-based sports, are diagnosed with mid-portion AT, following a clinical examination by a sports medicine physician and confirmed with MRI, and have been experiencing symptoms for more than 3 months but less than 3 years. 


\section{Exclusion criteria}

Patients will be ineligible for the study if they have a coexisting lower-limb injury, have had a running-related injury in the previous 12 months, or have had any peritendinous, or intra tendinous Achilles injection in the past 6 months, or previous Achilles surgery [15, 18, 44].

\section{Randomisation and blinding}

Participants will be assessed at baseline before being randomly assigned to the intervention group or control group and will follow a prescribed rehabilitation program for 12 weeks. See Table 3 for a summary of the study design. The randomisation will be performed using the online tool www.sealedenvelope.com and the participant will be handed an envelope from an independent observer not involved with the study, containing their respective group allocation number. The principal investigator and training group investigators will be blinded to the group randomisation process. These procedures have been used in similar studies [45]. The participants will be prescribed with an exercise program with video demonstrations of each exercise under the supervision of the investigator in their respective group. The program will be carried out at home or in a local gym in addition to supervised rehabilitation sessions every $2-3$ weeks at SSC. Testing will take place again at week 6 and 12 . Follow-up interviews will take place at 6,12 and 24 months after baseline testing. The investigator involved with the testing and follow-up interviews will also be blinded to the group allocation. The primary outcome measure will be changes to the VISA-A questionnaire. Secondary outcome measures will include plantar flexor strength, lower limb reactive strength, biomechanics and running gait.

\section{Outcome measures and assessments Investigations}

At baseline, week 6 and 12, all participants will be required to complete a VISA-A questionnaire as well as perform isokinetic testing and $3 \mathrm{D}$ motion capture running gait assessment. In addition, hop testing will be performed at week 6 and 12 [see Table 3]. Hop testing is included in the testing battery from week 6 onwards as it is expected some participants with Achilles pain at baseline testing may be fearful of performing hopping tasks or risk of exacerbating their pain, and the data collected may not be an accurate reflection of their capabilities.

\section{Primary outcome measure \\ VISA-A questionnaire}

The VISA-A questionnaire has been shown to be a valid, reliable and easy-to-use outcome measure tool for intervention studies on AT [46]. It consists of eight questions regarding pain and function during both daily living and sporting activities. The overall score is between 0 and 100 where higher scores represent reduced pain and improved function. An improvement of 21 points between 2 and 12 weeks of a rehabilitation program have been typically observed [44]. While the VISA-A score will not determine eligibility for inclusion into the study, it will be used to map progress over the course of the rehabilitation program and in the follow-up period. The difference in VISA-A score between both training protocols from baseline testing to the outcome testing at 6 weeks, 12 weeks, 3,6 and 12 months; will formulate the primary outcome measure for this study.

\section{Secondary outcome measures}

Isokinetic plantar flexor strength

Reduced plantar flexor strength is a common feature in patients with Achilles tendinopathy [14-16, 39]. One

Table 3 Overview of outcome measures over the course of the study

\begin{tabular}{|c|c|c|c|c|c|c|}
\hline & Baseline & Week 6 & Week 12 & 6 months & 12 months & 24 months \\
\hline Body mass & $x$ & & & & & \\
\hline Body height & $x$ & & & & & \\
\hline Body mass index & $x$ & & & & & \\
\hline Sport/activity level & $x$ & & & $x$ & $x$ & $X X$ \\
\hline VISA-A Questionnaire & $x$ & $x$ & $x$ & $x$ & $x$ & $x$ \\
\hline Isokinetic plantar flexor peak torque (knee extension) & $x$ & $x$ & $x$ & & & \\
\hline Isokinetic plantar flexor peak torque (knee flexion) & $x$ & $x$ & $x$ & & & \\
\hline $3 \mathrm{D}$ running gait analysis & $x$ & $x$ & $x$ & & & \\
\hline Double leg drop jump & & $x$ & $x$ & & & \\
\hline Single leg drop jump & & $x$ & $x$ & & & \\
\hline Single leg horizontal rebound & & $x$ & $x$ & & & \\
\hline Exercise compliance & & $x$ & $x$ & $x$ & & \\
\hline
\end{tabular}


prospective study to date established that plantar flexor torque below $50 \mathrm{Nm}$ was a risk factor for developing AT [47]. Isokinetic testing is commonly used to measure plantar flexor peak torque $[15,16,39]$.

Two separate protocols will be used for this test. In the first protocol, the participant will lie prone with full knee extension. In the second protocol, the participant will lie supine with $80^{\circ}$ knee flexion. When the knee is flexed to greater than $60^{\circ}$, the force contribution of the biarticular gastrocnemius muscles to plantarflexion is reduced, and is thus representative of the force produced predominantly by soleus muscle [48]. If similar plantar flexor peak torque deficits exist between the two protocols, the identified deficits may thus be attributed to the soleus muscle [15] which will influence exercise prescription.

The testing will be performed on a isokinetic dynamometer (Cybex Norm, Computer Sports Medicine Inc.). In both protocols, the participant will have their foot strapped to a pedal with the centre of axis of rotation aligned with the medial malleolus and a correction for gravity applied. Beginning with their uninjured limb, participants will be asked to perform a warm-up involving 5 sub maximal concentric plantarflexion and dorsiflexion contractions increasing progressively from 60 to $100 \%$ of their self-perceived MVC for familiarisation. The participants will then be required to produce a maximal plantarflexion force over 5 repetitions for 2 sets with a 1 min rest between sets. Verbal encouragement will be provided to produce maximal effort through full range of motion for each repetition. In the second test, the participants will lie in supine position with the knee flexed to $80^{\circ}$ in order to specifically test the peak torque of the soleus. The same familiarisation protocol, sets and repetitions as the previous test will apply. Both tests will use an angular velocity of $60^{\circ}$ per second and operate through an ankle range of between $30^{\circ}$ plantarflexion and $20^{\circ}$ dorsiflexion. Data will be sampled at 100 and peak torque expressed as percentage of body mass $(\mathrm{Nm} /$ $\mathrm{kg} \%$ ) will be reported on both limbs. Between-limb asymmetries in peak torque will also reported and analysed.

\section{Three-dimensional running gait analysis}

Altered running biomechanics and muscle recruitment strategies have been highlighted in runners with AT [49-52]. Using a proprietary three-dimensional optical motion analysis system (Run 3D, Oxford, United Kingdom) the following kinematic and spatiotemporal variables will be measured: contact time, aerial time, stride length, stride frequency and joint angular displacements from initial contact to mid stance phase. Lower limb stiffness will be calculated using a validated equation based on the spring-mass model with running speed, contact time, body mass and leg length as inputs [53]. The participants will warm-up by running for between 2 and $5 \mathrm{~min}$ on the treadmill at a self-selected speed. Once they report that they are adequately warmed up they will be instructed to run at a speed that they feel they would be comfortable running at a steady pace for $30 \mathrm{~min}$. Data will be captured for $30 \mathrm{~s}$ at a random interval over a 2 min period and the participants will not be informed about when the data capture begins. For the subsequent tests at week 6 and 12, the participants will be required to repeat the same speed for re-analysis.

\section{Hop tests}

Achilles tendon material properties contribute to stretch shortening cycle performance during hopping and jumping exercises $[54,55]$. Reduced tendon mechanical properties, plantar flexor muscle rate of force development and deficits on a single forward hop test have previously been observed in patients with AT [23].

The hop tests will take place on two force platforms (AMTI, USA) to measure ground reaction force (GRF) data sampled at $1000 \mathrm{~Hz}$. Ten infrared cameras $(200 \mathrm{~Hz}$; Bonita B10/Vero v2.2, Vicon, UK) will be used for threedimensional motion capture. Reflective markers $(14 \mathrm{~mm}$ diameter) placed on all relevant anatomical landmarks including the thorax, will be used in accordance with a modified Plug-in-gait model (Vicon, UK) [56], with centre of mass (COM) calculated from all segments. Motion and force data will be filtered using a fourth order zero-lag low pass Butterworth filter with a cut-off frequency of $15 \mathrm{~Hz}$. The data will be exported to MATLAB 2015a (Mathworks, USA) for processing. Participants will perform 3 trials on each test, unshod and with hands placed on iliac crests.

Drop jump Participants will perform both a double leg drop jump (DLDJ) and single leg drop jumps (SLDJ). The participants will complete a standardised warm-up which consists of 10 bodyweight squats, followed by 10 pogo hops in place and 3 familiarisation trials for both. A $30 \mathrm{~cm}$ box will be used for the DLDJ and a $20 \mathrm{~cm}$ box for the SLDJ. The participant who will be unshod with hands placed on iliac crests, will be required to drop off the box and rebound off the force plate as quickly as possible aiming for maximum jump height. They will be instructed to maintain knee and hip extension during flight phase and where there is visible evidence of knee flexion or a 'tuck jump', the trial will be deemed invalid and they will be asked to repeat until a competent trial is achieved. The ground contact phase will be defined by a GRF greater than $20 \mathrm{~N}$ and jump height will be calculated from centre of mass displacement using kinematic data. Reactive strength index (RSI), which is a measure 
of jump height divided by ground contact time, will be calculated for both the double and single leg drop jump.

Single leg hurdle hop After completing the drop jump tests, participants will be asked to perform a single leg forward hurdle hop test (SLHH). The test requires that participants to perform a single leg forward hop over two $15 \mathrm{~cm}$ hurdles rebounding off the force platform in between, completing 3 trials on each leg. The participants will be instructed to rebound 'as fast as possible' and 'as far as possible', and to attempt to be fully stable on 1 leg upon landing. After each trial the participants will walk back slowly to begin the next trial taking approximately $10 \mathrm{~s}$ recovery time. Hop distance, rebound distance and contact time, as well as key biomechanical variables such as vertical, horizontal and leg ground reaction force, vertical, leg and joint stiffness, joint powers and moments, and joint angular displacements will be calculated using a custom MATLAB script (Mathworks, USA). Hop distance will be calculated as the distance from the initiation of the hop to the initial contact as the participants lands at the end of the hop and rebound distance from the force plate to the landing. Vertical stiffness $\left(\mathrm{K}_{\mathrm{vert}}\right)$ will be calculated at the point of maximum displacement of COM, as the ratio of change in vertical ground reaction force (GRF) to COM displacement:

$$
\mathrm{K}_{\mathrm{vert}}=\Delta \text { Force } / \Delta \mathrm{CoM}
$$

Leg stiffness will be calculated in the sagittal plane as the ratio of change in leg ground reaction force $\left(\mathrm{F}_{\text {leg }}\right)$ to the change in leg length at the shortest leg length during stance phase as previously proposed [57]. Leg length is measured as the distance from the hip joint centre to the centre of pressure in the sagittal plane, while $F_{\text {leg }}$ is calculated from the resultant GRF magnitude scaled to the leg angle using the trigonometry sine rule.

$$
\mathrm{K}_{\text {leg }}=\Delta \mathrm{F}_{\text {leg }} / \Delta \mathrm{Leg}
$$

Joint stiffness ( $K_{\text {ankle, }} K_{\text {knee }}$ and $K_{h i p)}$ at the ankle and knee, will be calculated in the sagittal plane as the ratio of change in joint moment to change in joint displacement:

$$
\mathrm{K}_{\text {joint }}=\Delta \text { moment } / \Delta \text { angle }
$$

A pilot study has previously been carried out on 10 healthy participants prior to the commencement of the Achilles RCT study [58]. Good-to-excellent reliability (ICC > 0.75) was found for hop and rebound distance, contact time, knee and ankle joint stiffness, vertical and leg GRF, with moderate reliability (ICC 0.50-0.75) for reactive strength index, vertical and leg stiffness, ankle joint peak power, ankle and knee joint peak moments, and horizontal GRF. In a separate study using the same protocol, 3 trials were sufficient to obtain a stable measure of performance across key variables [59].

\section{Training diary}

In order to determine adherence and fidelity with the rehabilitation program and pain response to exercise, each patient will be required to complete a training diary logging their completed running and rehabilitation sessions as well as reporting any pain symptoms using a numerical pain rating scale (NPRS), that will be reviewed at week 6 and returned at week 12. Adherence is defined as the proportion of prescribed exercises completed while fidelity refers to whether the participant completed the prescribed exercises, sets, repetitions and target loads. Participants will be advised to take an extra recovery day between exercise sessions if pain was above $5 / 10$ on the day after a session and to adjust their loads for the subsequent session.

\section{Follow-up interviews}

At 6, 12 and 24 months from baseline testing, patients will be required to complete a questionnaire [see Additional file 1: Appendix 1] to analyse their participation in their respective sport, document any re-injuries and to obtain patient satisfaction feedback on their respective rehabilitation program. These outcomes will be reported and compared between groups to determine if the rehabilitation program had any significant effect (Fig. 1).

\section{Interventions}

Each participant will be prescribed a graded rehabilitation exercise training program which they will perform at home or in a local gym. However, they will present themselves for one supervised session every 2-3 weeks by their respective group investigators to ensure compliance and appropriate progression. The patients following Silbernagel's training program [see Table 1] will perform solely calf strength exercises with self-prescribed additional resistance and will progress their exercises based on a NPRS, where there is no greater than a $4 / 10$ pain response during and in the $24 \mathrm{~h}$ following a training session. They will then progress to plyometric exercises as tolerated. The SSC6 group [see Table 2] will follow a multifactorial exercise program comprising of bilateral and unilateral kinetic chain strength, calf strength and plyometric training as well as running drills as early as they can tolerate them. The participants will enter at the highest level where they meet the minimum criteria. For the calf exercises, they will be encouraged to lift a certain percentage of bodyweight in additional resistance and increase weekly. A certain level of pain within tolerable limits will be accepted and participants will be encouraged to increase their resistance loading weekly so 
long as that pain doesn't increase. Progression to Level 4 of the program will be based on achieving their prescribed exercise load targets and achieving a deficit of less than $10 \%$ between injured and uninjured limbs on the isokinetic strength tests. The reactive strength exercises will be performed at near maximal intensity for a set number of repetitions with good competency and within tolerable pain limits. The participants will progress to Level 5 when they can perform 10 single leg hops with a score of $<4 / 10$ on the NPRS and progress from Level 5 when single leg RSI deficits are $<10 \%$. Outcome measures will be monitored at the various timepoints and will be tracked according to reported NPRS ratings. In both groups, participants will be permitted to begin running in phase 2 when pain during daily activity is $<2 / 10$ but will be advised on periodising their running and rehabilitation exercises throughout the week. Each participant will be provided with a training log in order to monitor training loads. Should an adverse event occur which results in re-injury or a new injury, the participant will be instructed to contact their respective investigator immediately so that they can be examined and their treatment will be adjusted, postponed or discontinued where appropriate. Upon completion of the training intervention, participants in both groups will be given a maintenance training program for 6 months. The design, prescription and reporting of the training intervention meets all of the 16-item checklist requirements in the Consensus on Exercise Reporting Template (CERT) [60] [Available in supplementary files].
See Tables 1 and 2 for an example of the exercise programs, and Table 4 for the points of difference between to two training interventions.

\section{Statistical analysis and power calculation}

This study is powered to detect a change of 15 points on the VISA-A questionnaire, similar to previous studies investigating clinical changes after a rehabilitation program [61, 62]. The average reported change in a VISA-A questionnaire after a 12-week intervention is 21 points with a standard deviation 6.6 points [44]. Assuming a power of $80 \%$ and a two-sided $5 \%$ significance level, a total of 25 participants in each group would be required. Allowing for a conservative drop-out rate of $15 \%$, the proposed total sample size is 60 , with 30 in each group. This number is similar to those used in other high quality injury rehabilitation RCT studies [40, 45].

Statistical analysis will be performed using R (R Studio version 1.2.5). Descriptive statistics will be used for all continuous variables, and means and standard deviations will be reported. Comparisons between both groups at different timepoints will be assessed using Student's independent samples two-tailed t-tests. An intention to treat analysis will be used to test a within-group and between-group change in VISA-A questionnaire score at testing and follow-up timepoints, using a repeated measures Analysis of Covariance (ANCOVA). The primary outcome measure - changes to the VISA-A questionnaire, will the dependent variable, group will the between participants factor and time will be the covariate.

Table 4 SSC6 Versus Silbernagel's rehabilitation program.

\section{ssc6}

$\sqrt{2}$

Multi-factorial approach with emphasis on exercise progression, running gait and training load

$\sqrt{2}$

3 heavy load resistance training sessions per week with $>48 \mathrm{hrs}$ rest

(1)

Resistance load targets relative to body weight

Running gait re-training

\section{Silbernagel Combined}

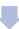

Emphasis on exercise progression based solely on symptoms

V.

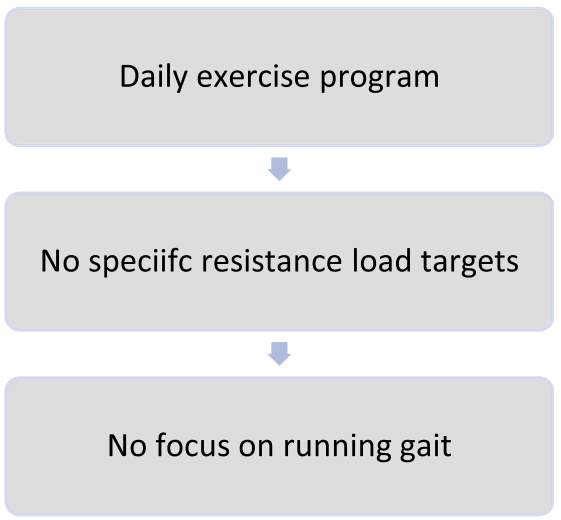


Non-parametric equivalents (Matt-Whitney U-Test and Friedman Test respectively) will be used if a ShapiroWilk test indicates that the assumption of normality has not been met. A multiple regression analysis will be used to investigate the total variance and the relative weight of each independent variable with changes in VISA-A score as the dependent variable. The independent variables will be changes in plantar flexor strength, reactive strength and lower limb biomechanical variables, as well as exercise adherence and fidelity. Effect sizes will be reported using partial eta squared threshold values of $>0.2$ (small), $>0.5$ (moderate), and $>0.8$ (large). Statistical significance will be accepted at $\alpha=0.05$.

\section{Discussion}

Exercise therapy is widely accepted as the primary treatment option for runners with AT [35, 36, 63]. Heavy resistance strength exercises targeting the muscle-tendon unit have been shown to increase physiological crosssectional area and pennation angle in the muscle [64] and tendon mechanical and material properties [41, 65]. This type of loading has resulted in improved clinical outcomes in AT patients [40,44]. Plyometric training prepares the muscle tendon unit for high tensile forces and loading rates associated with running based sports $[2,36,66]$. However, there is no clear guidance on how to prescribe and progress the loading for calf strength exercises apart from using pain response to exercise. Only a few studies have investigated running biomechanical features associated with AT with limited evidence for poor control of rearfoot eversion [50,67] and reduced leg stiffness on the injured limb [24].

An acceptable level of pain symptoms are permitted during AT rehabilitation [18, 34] and it remains to be explored if a primary focus on achieving strength, reactive strength and biomechanical targets can lead to similar outcomes in reduced timeframes and with lower reinjury rates. This is the first study to propose an individualised, criteria-based graded rehabilitation program in patients in with chronic mid-portion Achilles tendinopathy where progression is guided by strength and reactive strength outcome measures within tolerable pain limits. The participants in our study will undertake a comprehensive assessment of kinematic and kinetic tests to investigate plantar flexor strength, reactive strength and lower limb biomechanical variables in hopping and running. We will perform long term evaluations at evaluations at 6,12 and 24 months to monitor progress, reinjury incidences and sustainability of return to sport and investigate patient satisfaction with their respective rehabilitation exercise programs.

Our study will include a sample of participants who practise running-based sports and are of a particular age profile (age 18-45), have had no injection therapies in the previous 6 months and no co-existing lower limb injuries. Like most studies of similar design, there is a high risk of drop-outs, poor compliance with the respective programs and failure to respond to the follow-up questionnaires. This will be managed by aiming for a higher number of participants than the study is powered for and maintaining regular communication with the participants.

In summary, this two-arm RCT will compare the effectiveness of a criteria-based rehabilitation program with progression guided by achieving functional outcome measures with an evidenced-based program where progression is guided solely by pain symptoms. The results of this study will provide insights as to whether improved strength, reactive strength and lower limb biomechanics are associated with reduced pain in patients with chronic mid-portion AT and assist clinicians treating this injury to set objective criteria to progress rehabilitation and return to sport.

\section{Trial status}

Recruitment for the trial started in January 2020 and it is anticipated that data collection will be completed in April 2023. As of November 9th 2020, 18 participants have been included.

\section{Abbreviations}

ANCOVA: Analysis of covariance; AT: Achilles tendinopathy; CERT: Consensus on Exercise Reporting Template; COM: centre of mass; DLDJ: double leg drop jump; GRF: ground reaction force; ICC: intraclass correlation; K: stiffness; MRI: magnetic resonance image; MVC : maximal voluntary contraction; NPRS: numerical pain rating scale; NSAID: non-steroidal anti-inflammatory drug; RCT: randomised controlled trial; RSI: reactive strength index; SLDJ: single leg drop jump; SLHH: single leg hurdle hop; SPIRIT: Standard Protocol Items: Recommendations for Interventional Trials; SSC: Sports Surgery Clinic; VISA-A: Victorian Institute of Sports Assessment - Achilles

\section{Supplementary Information}

The online version contains supplementary material available at https://doi. org/10.1186/s12891-021-04553-6.

Additional file 1. Appendix 1: Follow-up questionnaire at 6 months. Additional file 2. Appendix 2: Consent form.

\section{Acknowledgements}

The authors would like to thank all participants, staff and referring physicians in advance for their help and support during this study.

\section{Related articles}

None declared.

\section{Authors' contributions}

CG, AFM and JBM devised the study design and protocol, and CG is leading the coordination of the study and investigations. KD provided biomechanical lab, statistical and data analysis support. CG and $\mathrm{CH}$ are investigators for respective training groups. CG wrote the manuscript and all authors provided feedback, input and final approval.

\section{Authors' information}

CG is a strength and conditioning coach specialising in the rehabilitation of lower limb injuries at the Sports Surgery Clinic in Dublin and is a PhD 
student affiliated to the Laboratory of Human Movement Science (LAMHESS) at Université Côte d'Azur in France.

KD is a Senior Lecturer in biomechanics at the Department of Sport and Exercise Sciences in Manchester Metropolitan University and was formerly manager of the biomechanics laboratory at the Sports Surgery Clinic in Dublin (2015-2020)

$\mathrm{CH}$ is a physiotherapist at the Sports Surgery Clinic in Dublin.

AFM is a sport and exercise medicine physician and Director of Sports Medicine at the Sports Surgery Clinic in Dublin.

JBM is a Professor at Université Jean Monnet, Saint-Etienne, France and previously at the Laboratory of Human Movement Science (LAMHESS) at Université Côte d'Azur in France (2014-2020).

\section{Funding}

This study is internally funded and supported by the Sports Surgery Clinic, Dublin (SSC). The SSC were involved in the planning and design of the study.

\section{Availability of data and materials}

Data sharing is not applicable to this article as no datasets were generated or analysed during the current study.

\section{Declarations}

\section{Ethics approval and consent to participate}

Ethical approval was obtained by the Sports Surgery Clinic Research and Ethics Committee (Approval number: SAREB13/05/19CG/MJ). Participants will sign written informed consent prior to taking part in the study.

\section{Consent for publication}

Not applicable.

\section{Competing interests}

None declared.

\section{Author details}

${ }^{1}$ Université Côte d'Azur, LAMHESS, Nice, France. ${ }^{2}$ Sports Surgery Clinic, Santry Demesne, Dublin 9, Ireland. ${ }^{3}$ University of Bristol, Queen's School of Engineering, University Walk, Bristol BS81TR, UK. ${ }^{4}$ Department of Sport and Exercise Sciences, Manchester Metropolitan University, Manchester, UK. ${ }^{5}$ Centre for Health, Exercise and Sports Medicine, University of Melbourne, Parkville, Victoria, Australia. ${ }^{6}$ Sports Performance Research Institute New Zealand (SPRINZ), Auckland University of Technology, Auckland, New Zealand. 'Univ Lyon, UJM-Saint-Etienne, Inter-university Laboratory of Human Movement Biology, EA 7424, F-42023 Saint-Etienne, France.

Received: 14 January 2021 Accepted: 7 July 2021

Published online: 14 August 2021

\section{References}

1. Harris CA, Peduto AJ. Achilles tendon imaging. Australas Radiol. 2006;50(6): 513-25. https://doi.org/10.1111/j.1440-1673.2006.01622.x.

2. Komi $P$ V. Relevance of in vivo force measurements to human biomechanics. J Biomech. 1990;23(SUPPL. 1):23-34.

3. Wren TAL, Yerby SA, Beaupré GS, Carter DR. Mechanical properties of the human achilles tendon. Clin Biomech. 2001;16(3):245-51. https://doi.org/10.1 016/S0268-0033(00)00089-9.

4. De Jonge S, Van Den Berg C, De Vos RJ, Van Der Heide HJL, Weir A, Verhaar JAN, et al. Incidence of midportion Achilles tendinopathy in the general population. Br J Sports Med. 2011;45(13):1026-8. https://doi.org/10.1136/ bjsports-2011-090342.

5. Kujala UM, Sarna S, Kaprio J. Cumulative incidence of achilles tendon rupture and tendinopathy in male former elite athletes. Clin J Sport Med. 2005;15(3):133-5. https://doi.org/10.1097/01.jsm.0000165347.55638.23.

6. Cook JL, Khan KM, Purdam C. Achilles tendinopathy. Man Ther. 2002;7(3): 121-30. https://doi.org/10.1054/math.2002.0458.

7. Khan KM. Time to abandon the "tendinitis" myth. Bmj [Internet]. 2002; 324(7338):626-7. Available from:. https://doi.org/10.1136/bmj.324.7338.626.

8. Cook JL, Purdam CR. Is tendon pathology a continuum? A pathology model to explain the clinical presentation of load-induced tendinopathy. $\mathrm{Br} \mathrm{J}$ Sports Med. 2009;43(6):409-16. https://doi.org/10.1136/bjsm.2008.051193.
9. Jones GC, Corps AN, Pennington CJ, Clark IM, Edwards DR, Bradley MM, et al. Expression profiling of metalloproteinases and tissue inhibitors of metalloproteinases in normal and degenerate human Achilles tendon. Arthritis Rheum. 2006;54(3):832-42. https://doi.org/10.1002/art.21672.

10. Riley G. Tendinopathy - from basic science to treatment. Nat Clin Pract Rheumatol. 2008;4(2):82-9. https://doi.org/10.1038/ncprheum0700.

11. Selvanetti A, Cipolla M, Puddu G. Overuse tendon injuries: basic science and classification. Oper Tech Sports Med [Internet]. 1997;5(3):110-7. Available from: http://linkinghub.elsevier.com/retrieve/pii/S1060187297800317. https:// doi.org/10.1016/S1060-1872(97)80031-7.

12. Kjær M, Langberg H, Heinemeier K, Bayer ML, Hansen M, Holm L, et al. From mechanical loading to collagen synthesis, structural changes and function in human tendon. Scand J Med Sci Sport. 2009;19(4):500-10. https://doi. org/10.1111/j.1600-0838.2009.00986.x.

13. Mead MP, Gumucio JP, Awan TM, Mendias CL, Sugg KB. Pathogenesis and management of tendinopathies in sports medicine. Transl Sport Med [Internet]. 2018;1(1):5-13. Available from:. https://doi.org/10.1002/tsm2.6.

14. Silbernagel KG, Gustavsson A, Thomeé R, Karlsson J. Evaluation of lower leg function in patients with Achilles tendinopathy. Knee Surg Sport Traumatol Arthrosc. 2006;14(11):1207-17. https://doi.org/10.1007/s00167-006-0150-6.

15. O'Neill S, Barry S, Watson P. Plantarflexor strength and endurance deficits associated with mid-portion Achilles tendinopathy: the role of soleus. Phys Ther Sport [internet]. 2019;37:69-76. Available from: https://doi.org/10.1016/ j.ptsp.2019.03.002.

16. McAuliffe S, Tabuena A, McCreesh K, O'Keeffe M, Hurley J, Comyns T, et al. Altered Strength Profile in Achilles Tendinopathy: A Systematic Review and Meta-Analysis. J Athl Train. 2019;54(8):889-900.

17. Järvinen TAH, Kannus P, Maffulli N, Khan KM. Achilles tendon disorders: etiology and epidemiology. Foot Ankle Clin. 2005;10(2):255-66. https://doi. org/10.1016/j.fcl.2005.01.013

18. Silbernagel KG, Thomeé R, Eriksson BI, Karlsson J. Continued sports activity, using a pain-monitoring model, during rehabilitation in patients with achilles tendinopathy: a randomized controlled study. Am J Sports Med. 2007;35(6):897-906. https://doi.org/10.1177/0363546506298279.

19. Silbernagel KG, Thomeé R, Eriksson BI, Karlsson J. Full symptomatic recovery does not ensure full recovery of muscle-tendon function in patients with Achilles tendinopathy. Br J Sports Med. 2007;41(4):276-80. https://doi.org/1 0.1136/bjsm.2006.033464.

20. Knobloch K, Schreibmueller L, Longo UG, Vogt PM. Eccentric exercises for the management of tendinopathy of the main body of the Achilles tendon with or without an AirHeel ${ }^{\mathrm{TM}}$ brace. A randomized controlled trial. B: effects of compliance. Disabil Rehabil. 2008;30(20-22):1692-6. https://doi.org/10.1 080/09638280701785676.

21. Arya S, Kulig K. Tendinopathy alters mechanical and material properties of the Achilles tendon. J Appl Physiol [Internet]. 2010;108(3):670-5. Available from:. https://doi.org/10.1152/japplphysiol.00259.2009.

22. Child S, Bryant AL, Clark RA, Crossley KM. Mechanical properties of the achilles tendon aponeurosis are altered in athletes with achilles tendinopathy. Am J Sports Med. 2010;38(9):1885-93. https://doi.org/10.1177/ 0363546510366234.

23. Wang HK, Lin KH, Su SC, Shih TTF, Huang YC. Effects of tendon viscoelasticity in Achilles tendinosis on explosive performance and clinical severity in athletes. Scand J Med Sci Sport. 2012;22(6):1-9.

24. Maquirriain J. Leg stiffness changes in athletes with achilles tendinopathy. Int J Sports Med. 2012;33(7):567-71. https://doi.org/10.1055/s-0032-1304644.

25. Martin RL, Chimenti R, Cuddeford Tyler, Houck J, Matheson JW, Mcdonough CM, et al. Clinical Practice Guidelines Achilles Pain, Stiffness, and Muscle Power Deficits: Midportion Achilles Tendinopathy Revision 2018 SUMMARY OF RECOMMENDATIONS. J Orthop Sport Phys Ther [Internet]. 2018 [cited 2020];48(5):1-38. Available from: www.jospt.org

26. Kearney RS, Parsons N, Metcalfe D, Costa ML. Injection therapies for Achilles tendinopathy [Internet]. Vol. 2015, Cochrane Database of Systematic Reviews. John Wiley and Sons Ltd; 2015 [cited 2020 Jul 29]. Available from: https://doi.org/10.1002/14651858.CD010960.pub2/full

27. Chimenti RL, Cychosz CC, Hall MM, Phisitkul P. Current Concepts Review Update: Insertional Achilles Tendinopathy. Foot Ankle Int [Internet]. 2017 [cited 2020 Jul 29];38(10):1160-1169. Available from: https://www.ncbi.nlm. nih.gov/pmc/articles/PMC5956523/

28. Rompe JD, Nafe B, Furia JP, Maffulli N. Eccentric loading, shock-wave treatment, or a wait-and-see policy for tendinopathy of the main body of tendo Achillis: a randomized controlled trial. Am J Sports Med [Internet]. 
2007 Mar 30 [cited 2020 Jul 29];35(3):374-383. Available from: http://www. ncbi.nlm.nih.gov/pubmed/17244902.

29. Magnussen RA, Dunn WR, Thomson AB. Nonoperative treatment of midportion achilles tendinopathy: a systematic review. Clin J Sport Med. 2009;19(1):54-64. https://doi.org/10.1097/JSM.0b013e31818ef090.

30. Zhang BM, Zhong LW, Xu SW, Jiang HR, Shen J. Acupuncture for chronic achilles tendnopathy: A randomized controlled study. Chin J Integr Med [Internet]. 2013 [cited 2020 Jul 29];19(12):900-904. Available from: https:// doi.org/10.1007/s11655-012-1218-4

31. De Jonge S, De Vos RJ, Van Schie HTM, Verhaar JAN, Weir A, Tol JL. Oneyear follow-up of a randomised controlled trial on added splinting to eccentric exercises in chronic midportion Achilles tendinopathy. Br J Sports Med [Internet]. 2010 [cited 2020 Jul 29];44(9):673-677. Available from: https://bjsm.bmj.com/content/44/9/673

32. Romero-Morales C, Martín-Llantino PJ, Calvo-Lobo C, Beltran-Alacreu H, López-López D, Sánchez-Gómez R, et al. Effectiveness of eccentric exercise and a vibration or cryotherapy program in enhancing rectus abdominis muscle thickness and inter-rectus distance in patients with chronic midportion Achilles tendinopathy: a randomized clinical trial. Int J Med Sci [Internet]. 2018;15(14):1764-70. Available from: http://www.medsci.org/v1 5p1764.htm. https://doi.org/10.7150/ijms.28656.

33. Balius R, Álvarez G, Baró F, Jiménez F, Pedret C, Costa E, et al. A 3-Arm Randomized Trial for Achilles Tendinopathy: Eccentric Training, Eccentric Training Plus a Dietary Supplement Containing Mucopolysaccharides, or Passive Stretching Plus a Dietary Supplement Containing Mucopolysaccharides. Curr Ther Res [Internet]. 2016;78:1-7 Available from: https://linkinghub.elsevier.com/retrieve/pii/S0011393X16300716.

34. Cook JL, Purdam CR. The challenge of managing tendinopathy in competing athletes. Br J Sports Med. 2014;48(7):506-9. https://doi.org/1 0.1136/bjsports-2012-092078

35. Vlist AC Van Der, Winters M, Weir A, Ardern CL, Vos J De, Welton NJ, et al. Which treatment is most effective for patients with Achilles tendinopathy ? A living systematic review with network meta- analysis of 29 randomised controlled trials. 2020;45(11):1-8.

36. Silbernagel KG. A Proposed Return-to-Sport Program for Patients With Midportion Achilles Tendinopathy: Rationale and Implementation. J Orthop Sports Phys Ther. 2015;45(11):876-86. Available from: www.jospt.org

37. Lavagnino M, Wall ME, Little D, Banes AJ, Guilak F, Arnoczky SP. Tendon Mechanobiology: current knowledge and future research opportunities. J Orthop Res. 2015;33(6):813-22. https://doi.org/10.1002/jor.22871.

38. Snedeker JG, Foolen J. Tendon injury and repair - A perspective on the basic mechanisms of tendon disease and future clinical therapy. Acta Biomaterialia. 2017;63:18-36.

39. Alfredson $\mathrm{H}$, Pietila $\mathrm{T}$, Jonsson P, Lorentzon R. Heavy-load eccentric calf muscle training for the treatment of chronic Achilles tendinosis. Am J Sport Med [Internet]. 1998;26(3):360-6. Available from: http://ajs.sagepub.com/ content/26/3/360.short. https://doi.org/10.1177/03635465980260030301.

40. Beyer R, Kongsgaard M, Hougs Kjær B, Øhlenschlæger T, Kjær M, Magnusson SP. Heavy slow resistance versus eccentric training as treatment for achilles tendinopathy: a randomized controlled trial. Am J Sports Med. 2015:43(7):1704-11. https://doi.org/10.1177/0363546515584760.

41. Bohm S, Mersmann F, Arampatzis A. Human tendon adaptation in response to mechanical loading: a systematic review and meta-analysis of exercise intervention studies on healthy adults. Sport Med - Open. 2015;1 (1):1-7.

42. Rio E, Kidgell D, Purdam C, Gaida J, Moseley GL, Pearce AJ, et al. Isometric exercise induces analgesia and reduces inhibition in patellar tendinopathy. Br J Sports Med. 2015;49(19):1277-83. https://doi.org/10.1136/bjsports-2014094386.

43. O'Neill S, Radia J, Bird K, Rathleff MS, Bandholm T, Jorgensen M, et al. P23: acute sensory and motor response to 45-seconds heavy isometric holds for the plantar flexors in patients with Achilles tendinopathy. Online J Rural Nurs Heal Care [Internet]. 2017;17(1):SS20-0. Available from:. https://doi. org/10.1007/s00167-018-5050-z.

44. Murphy M, Travers M, Gibson W, Chivers P, Debenham J, Docking S, et al. Rate of Improvement of Pain and Function in Mid-Portion Achilles Tendinopathy with Loading Protocols: A Systematic Review and Longitudinal Meta-Analysis. Sport Med [Internet]. 201815 [cited 2018 Sep 10];48(8):1875-1891. Available from: https://doi.org/10.1007/s40279-0180932-2

45. Mendiguchia J, Martinez-Ruiz E, Edouard P, Morin JB, Martinez-Martinez F, Idoate F, et al. A multifactorial, criteria-based progressive algorithm for hamstring injury treatment. Med Sci Sports Exerc. 2017;49(7):1482-92. https://doi.org/10.1249/MSS.0000000000001241.

46. Robinson JM, Cook JL, Purdam C, et al. The VISA-A questionnaire: a valid and reliable index of the clinical severity of Achilles tendinopathy. $\mathrm{Br} J$ Sports Med. 2001;35:335-41.

47. Mahieu NN. Intrinsic risk factors for the development of Achilles tendon overuse injury: a prospective study. Am J Sports Med. 2006;34(2):226-35. https://doi.org/10.1177/0363546505279918.

48. Arampatzis A, Karamanidis K, Stafilidis S, Morey-Klapsing G, DeMonte G, Brüggemann GP. Effect of different ankle- and knee-joint positions on gastrocnemius medialis fascicle length and EMG activity during isometric plantar flexion. J Biomech. 2006;39(10):1891-902. https://doi.org/10.1016/j. jbiomech.2005.05.010.

49. Azevedo LB, Lambert MI, Vaughan $C L, O^{\prime}$ Connor CM, Schwellnus MP. Biomechanical variables associated with Achilles tendinopathy in runners. Br J Sports Med. 2009:43(4):288-92.

50. Munteanu SE, Barton CJ, Hootman J, Macera C, Ainsworth B, Addy C, et al. Lower limb biomechanics during running in individuals with achilles tendinopathy: a systematic review. J Foot Ankle Res [Internet]. 2011;4(1):15. Available from: https://doi.org/10.1186/1757-1146-4-15

51. Geremia JM, Bobbert MF, Casa Nova M, Ott RD, De Aguiar LF, De Oliveira $L R$, et al. The structural and mechanical properties of the Achilles tendon 2 years after surgical repair. Clin Biomech [Internet]. 2015;30(5):485-92. Available from:: https://doi.org/10.1016/j.clinbiomech.2015.03.005.

52. Ogbonmwan I, Kumar BD, Paton B. New lower-limb gait biomechanical characteristics in individuals with Achilles tendinopathy: A systematic review update. Gait Posture [Internet]. 2018 May [cited 2018 Sep 10];62:146-156. Available from: https://linkinghub.elsevier.com/retrieve/pii/S09666362183 01425

53. Morin JB, Dalleau G, Kyröläinen H, Jeannin T, Belli A. A simple method for measuring stiffness during running. J Appl Biomech. 2005;21(2):167-80. https://doi.org/10.1123/jab.21.2.167.

54. Kubo K, Kawakami Y, Fukunaga T, James RS, Navas CA, Herrel A. Influence of elastic properties of tendon structures on jump performance in humans influence of elastic properties of tendon structures on jump performance in humans. J Appl Physiol. 1999;87(6):2090-6. https://doi.org/10.1152/jappl.1 999.87.6.2090.

55. Abdelsattar M, Konrad A, Tilp M. Relationship between achilles tendon stiffness and ground contact time during drop jumps. J Sport Sci Med. 2018;17(2):223-8.

56. Marshall BM, Franklyn-Miller AD, King EA, Moran KA, Strike SC, Falvey ÉC. Biomechanical Factors Associated With Time to Complete a Change of Direction Cutting Maneuver. J Strength Cond Res [Internet]. 20141 [cited 2020 Nov 7];28(10):2845-2851. Available from: http://journals.lww.com/ 00124278-201410000-00019

57. Coleman DR, Cannavan D, Horne S, Blazevich AJ. Leg stiffness in human running: comparison of estimates derived from previously published models to direct kinematic-kinetic measures. J Biomech [Internet]. 2012;45(11):1987-91. Available from: https://doi.org/10.1016/j.jbiomech.2012.05.010.

58. Griffin C, Richter C, Franklyn-Miller A, Morin JB. A novel single-leg hurdle hop exercise: test-retest reliability of vertical stiffness, contact time, ground reaction force, total hop distance and rebound distance. World Congress of Biomechanics [Conference presentation]. 2018.

59. Griffin C, Richter C, Franklyn-Miller A, Morin JB. The number of trials required to obtain a true representation of biomechanical features extracted during a single leg hurdle hop exercise. European College of Sports Science [Conference Presentation]. 2018.

60. Slade SC, Dionne CE, Underwood M, Buchbinder R, Beck B, Bennell K, et al. Consensus on exercise reporting template (Cert): modified delphi study. Phys Ther. 2016;96(10):1514-24. https://doi.org/10.2522/ptj.20150668.

61. Rompe JD, Furia J, Maffulli N. Eccentric loading versus eccentric loading plus shock-wave treatment for midportion achilles tendinopathy: a randomized controlled trial. Am J Sports Med. 2009;37(3):463-70. https://doi. org/10.1177/0363546508326983.

62. Stevens $\mathrm{M}$, Tan C-W. Effectiveness of the Alfredson protocol compared with a lower repetition-volume protocol for Midportion Achilles tendinopathy: a randomized controlled trial. J Orthop Sport Phys Ther [Internet]. 2014;44(2): 59-67. Available from:. https://doi.org/10.2519/jospt.2014.4720.

63. Kountouris A, Cook J. Rehabilitation of Achilles and patellar tendinopathies. Best Pract Res Clin Rheumatol. 2007;21(2):295-316. https://doi.org/10.1016/j. berh.2006.12.003. 
64. Albracht $\mathrm{K}$, Arampatzis A. Exercise-induced changes in triceps surae tendon stiffness and muscle strength affect running economy in humans. Eur J Appl Physiol. 2013;113(6):1605-15. https://doi.org/10.1007/s00421-012-2 585-4.

65. Geremia JM, Manfredini B, Maarten B, Bobbert F, Rico R, Juner F, et al. Effects of high loading by eccentric triceps surae training on Achilles tendon properties in humans. Eur J Appl Physiol [Internet]. Eur J Appl Physiol. 2018;118(8):1725-36. Available from: https://doi.org/10.1007/s00421018-3904-1

66. Baxter JR, Corrigan P, Hullfish TJ, O'Rourke P, Silbernagel KG. Exercise Progression to Incrementally Load the Achilles Tendon. Med Sci Sports Exerc. 2021;53(1):124-30. https://doi.org/10.1249/MSS.0000000000002459.

67. Donoghue OA, Harrison AJ, Coffey N, Hayes K. Functional data analysis of running kinematics in chronic Achilles tendon injury. Med Sci Sports Exerc. 2008;40(7):1323-35. https://doi.org/10.1249/MSS.0b013e31816c4807.

\section{Publisher's Note}

Springer Nature remains neutral with regard to jurisdictional claims in published maps and institutional affiliations.

Ready to submit your research? Choose BMC and benefit from:

- fast, convenient online submission

- thorough peer review by experienced researchers in your field

- rapid publication on acceptance

- support for research data, including large and complex data types

- gold Open Access which fosters wider collaboration and increased citations

- maximum visibility for your research: over $100 \mathrm{M}$ website views per year

At $\mathrm{BMC}$, research is always in progress.

Learn more biomedcentral.com/submissions 